\title{
ChemComm
}

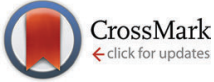

Cite this: Chem. Commun., 2015, 51, 13957

Received 8th July 2015, Accepted 27th July 2015

DOI: $10.1039 / c 5 c c 05651 a$

www.rsc.org/chemcomm

\section{ortho-(Methyltosylaminoethynyl)benzyl glycosides as new glycosyl donors for latent-active glycosylation $\dagger$}

\author{
Xiaoping Chen, ${ }^{a}$ Dacheng Shen, ${ }^{a}$ Qiaoling Wang, ${ }^{a}$ You Yang ${ }^{b}$ and Biao Yu*a
}

\begin{abstract}
A new glycosylation protocol employing ortho-(methyltosylaminoethynyl)benzyl glycosides as glycosyl donors and TMSOTf as the catalyst is disclosed. These donors can be readily prepared from the corresponding 'latent' ortho-iodobenzyl glycosides via a Sonogashira coupling, thus providing a new approach for the 'latent-active' synthesis of glycans.
\end{abstract}

New glycosylation methods are continuously developed with efforts to construct the extremely diverse glycosidic linkages occurring in glycans and glycoconjugates in an efficient and economical manner. ${ }^{1}$ In the last decade, much interest has been devoted to the investigation of glycosylation protocols based on activation of the $\mathrm{C} \equiv \mathrm{C}$ triple bonds. Thus, propargyl glycosides and 1,2-orthoesters (under the catalysis of $\mathrm{AuX}_{3}$ ), ${ }^{2,3}$ glycosyl alkynoates (under the promotion of $\left.\mathrm{Hg}(\mathrm{OTf})_{2}\right),{ }^{4}$ dipropargylcyanoacetates (under the catalysis of $\mathrm{AuCl}_{3} / \mathrm{AgSbF}_{6}$ ), ${ }^{5}$ as well as alkynyl-containing thioglycosides (under the catalysis of $\mathrm{Au}(\mathrm{I})$ complexes), ${ }^{6}$ are disclosed to be effective donors in certain glycosylation reactions. Especially, glycosyl ortho-alkynylbenzoates (under the catalysis of a gold(I) complex, such as $\mathrm{PPh}_{3} \mathrm{AuNTf}_{2}$ and $\mathrm{PPh}_{3} \mathrm{AuOTf}$ ) have been found to have wide applications in the synthesis of complex glycans and glycoconjugates; ${ }^{7,8}$ and the general mechanism of this glycosylation reaction has been largely elucidated. ${ }^{7-e}$ During the course of these studies, we tried glycosyl orthoalkynylbenzyl glycosides as donors, which would have the advantage of easy manipulation of the protecting groups, but found no glycosylation took place under similar conditions wherein the corresponding ortho-alkynylbenzoates underwent glycosylation. We envisioned introduction of an electron-rich substituent on the alkyne moiety to facilitate the desired glycosylation pathway. Here we report ortho-(methyltosylaminoethynyl)benzyl glycosides

\footnotetext{
${ }^{a}$ State Key Laboratory of Bio-organic and Natural Products Chemistry, Shanghai Institute of Organic Chemistry, Chinese Academy of Sciences, 345 Lingling Road, Shanghai 200032, China. E-mail: byu@mail.sioc.ac.cn

${ }^{b}$ Shanghai Key Laboratory of New Drug Design, School of Pharmacy, East China University of Science and Technology, 130 Meilong Road, Shanghai 200237, China $\dagger$ Electronic supplementary information (ESI) available. See DOI: 10.1039/ c5cc05651a
}

as a new type of glycosyl donors which can be activated by a catalytic amount of TMSOTf under mild conditions and their applicability in the 'latent-active' synthesis of glycans.

The desired glycosyl ortho-(methyltosylaminoethynyl)benzyl glycosides could be easily prepared from the corresponding ortho-iodobenzyl glycosides via a Sonogashira coupling with ynamide 4. $^{9-11}$ Taking the preparation of perbenzoyl glucopyranoside 1a as an example (Scheme 1), ortho-iodobenzyl glucopyranoside 3a was obtained via condensation of perbenzoylated glucose 2a with 2-iodobenzyl alcohol under the action of TMSOTf, which was then subjected to coupling with ynamide 4 in the presence of $\left(\mathrm{PPh}_{3}\right)_{2} \mathrm{PdCl}_{2}$ and $\mathrm{CuI}$ in $\mathrm{i}-\mathrm{Pr}_{2} \mathrm{NH} / \mathrm{DMF}$ to provide the desired ortho-(methyltosylaminoethynyl)benzyl glucoside 1a in high yield (90\%). ${ }^{10}$ Manipulation of the protecting groups on the iodobenzyl glycoside $\mathbf{3 a}$ followed by Sonogashira coupling would lead to (methyltosylaminoethynyl)benzyl glucopyranosides bearing different protecting group patterns, such as 1c (Table 2). (Methyltosylaminoethynyl)benzyl rhamnopyranoside 1b and 2-deoxy-glucopyranoside 1d were similarly prepared by Sonogashira coupling as the key step (see ESI $\dagger$ for details). All these glycosides were found to be stable when stored at room temperature.

With the perbenzoyl glucoside 1a as a potential donor and cholesterol 5a as an acceptor, a variety of $\pi$-acids and Lewis acids (0.1 eq.) were screened as promoters for the desired

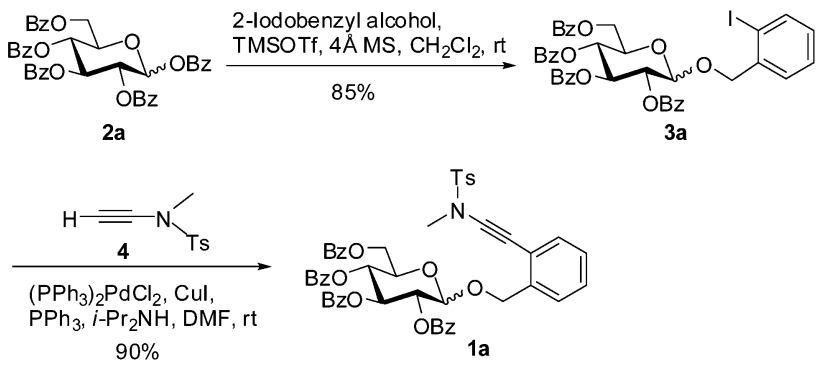

Scheme 1 Preparation of ortho-(methyltosylaminoethynyl)benzyl glucopyranoside 1a. 
Table 1 Screening of promoters for the glycosylation of ortho-(methyltosylaminoethynyl)benzyl glucoside 1 a with cholesterol $\mathbf{5 a}$

\begin{tabular}{|c|c|c|}
\hline OBz & 5a(1.0 eq.) & $\underbrace{}_{\mathrm{OBz}}$ \\
\hline Entry & Promoter (0.1 eq.) & Isolated yield (\%) \\
\hline 1 & $\mathrm{PPh}_{3} \mathrm{AuNTf}_{2}$ & 43 \\
\hline 2 & TMSOTf & 91 \\
\hline 3 & $\mathrm{Bi}(\mathrm{OTf})_{3}$ & 83 \\
\hline 4 & $\operatorname{In}(\mathrm{OTf})_{3}$ & 67 \\
\hline 5 & $\mathrm{Cu}(\mathrm{OTf})_{2}$ & 46 \\
\hline 6 & $\mathrm{Sc}(\mathrm{OTf})_{3}$ & 17 \\
\hline 7 & $\mathrm{BF}_{3} \mathrm{OEt}_{2}$ (1.2 eq.) & 21 \\
\hline 8 & $\mathrm{SnCl}_{4}$ & 26 \\
\hline 9 & $\mathrm{PtCl}_{2}$ & 16 \\
\hline 10 & $\mathrm{AuBr}_{3}$ & Trace \\
\hline 11 & CuI or LiOTf & No reaction \\
\hline
\end{tabular}

glycosylation reaction in the presence of $4 \AA \mathrm{MS}$ in $\mathrm{CH}_{2} \mathrm{Cl}_{2}$ at room temperature (Table 1). Surprisingly, $\mathrm{PPh}_{3} \mathrm{AuNTf}_{2}$, the effective catalyst for the glycosylation of glycosyl ortho-alkynylbenzoates, ${ }^{7}$ could not catalyze the present coupling effectively, providing the desired $\beta$-glucoside 6aa in only $43 \%$ yield (entry 1 ). The major byproduct arose from the nucleophilic addition of cholesterol 5a onto the ynamide moiety. ${ }^{11 a, e}$ TMSOTf turned out to be the most effective catalyst, wherein the coupled glycoside 6 aa was obtained in a high yield of $91 \%$ (entry 2). Bi(OTf $)_{3}, \operatorname{In}(\mathrm{OTf})_{3}$, and $\mathrm{Cu}(\mathrm{OTf})_{2}$ were shown to be better catalysts than $\mathrm{PPh}_{3} \mathrm{AuNTf}_{2}$, leading to $6 \mathbf{a a}$ in $83 \%, 67 \%$, and $46 \%$ yield, respectively (entries $3-5$ ). Sc(OTf) ${ }_{3}$, $\mathrm{BF}_{3} \mathrm{OEt}_{2}, \mathrm{SnCl}_{4}$, and $\mathrm{PtCl}_{2}$ were found to be ineffective for this coupling (16-26\%) (entries 6-9), whereas the coupling partners stayed inert in the presence of $\mathrm{AuBr}_{3}$, CuI, and LiOTf (entries 10 and 11).

Next, we investigated briefly the scope of the TMSOTf-catalyzed glycosylation reaction with ortho-(methyltosylaminoethynyl)benzyl glycosides as donors (Table 2). Four representative glycosides $\mathbf{1 a}-\mathbf{- 1 d}$ and four alcohols $\mathbf{5 a - 5 d}$ were selected as coupling partners, and all the reactions were carried out under fixed conditions (0.1 eq. TMSOTf, $4 \AA \mathrm{AS}, \mathrm{CH}_{2} \mathrm{Cl}_{2}, \mathrm{rt}, 2 \mathrm{~h}$ ). The couplings of perbenzoyl-glucopyranoside 1a with all the four alcohols led to the coupled glycosides in excellent yields $(>91 \%)$ and with complete $\beta$-selectivity (entries 1-4), testifying the participation of the neighbouring group in the glycosylation. ${ }^{1}$ Similarly, the couplings of 2,3,4-tri-O-benzoyl-L-rhamnopyranoside $\mathbf{1 b}$ with alcohols $\mathbf{5 a - 5 d}$ provided the corresponding $\alpha$-L-rhamnosides in a fully stereocontrolled manner in high yields (88-95\%; entries 5-8). As expected, the corresponding glycosylation reactions of perbenzylglucopyranoside 1c and 2-deoxy-glucopyranoside 1d, in the absence of a neighboring participating group, led to the coupled glycosides in high yields (83-99\%), albeit in a pair of the $\alpha$ - and $\beta$-anomers (Table 2, entries 11-16). ${ }^{12,13}$ It was noted that the reactions with the hindered glucose-4-OH derivative $5 \mathbf{c}$ as the acceptor were devoid of the addition of alcohol onto the ynamide moiety, therefore the unglycosylated $\mathbf{5 c}$ could be fully recovered.

In fact, the addition of alcohol onto the ynamide moiety became a serious problem when the alcohol to be glycosylated
Table 2 Glycosylation with ortho-(methyltosylaminoethynyl)benzyl glycosides $\mathbf{1 a - 1 d}$ as donors under the catalysis of TMSOTf

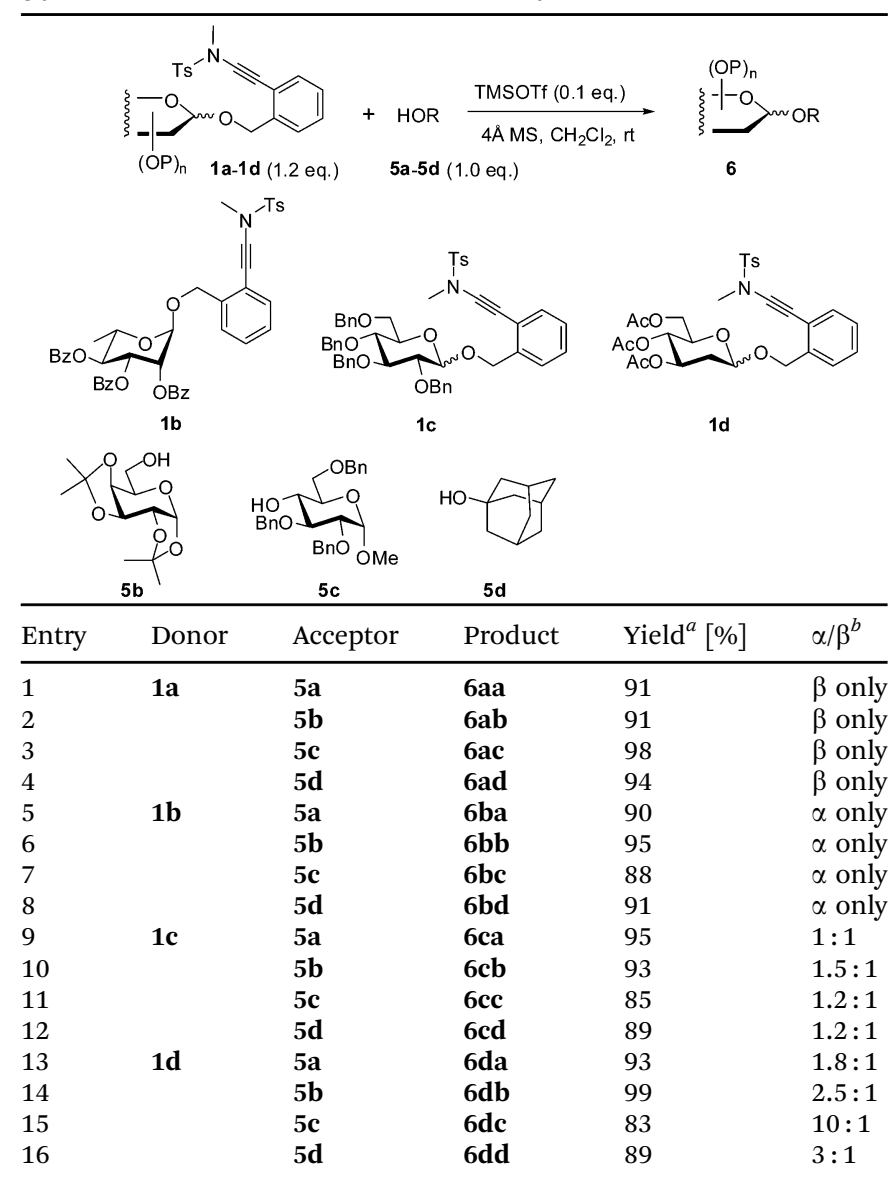

${ }^{a}$ Isolated yield. ${ }^{b}$ The $\alpha / \beta$ ratio was determined by ${ }^{1} \mathrm{H}$ NMR spectroscopic measurement.

is highly reactive. Thus, the condensation of 1a with 4-penten-1ol 5e under the catalysis of TMSOTf delivered the coupled glycoside 6 ae in only $62 \%$ yield, while ester 7 , which was derived from the corresponding adduct during workup, was isolated in $34 \%$ yield (Scheme 2). ${ }^{11}$

Based on these experimental findings and the nature of ynamides, ${ }^{9,11}$ a plausible mechanism for the present TMSOTfcatalyzed glycosylation reaction with ortho-(methyltosylaminoethynyl)benzyl glycosides as donors was proposed (Scheme 3). Thus, keteniminium cation $\mathbf{B}$ was generated from ortho-(methyltosylaminoethynyl)benzyl glycoside $\mathbf{A}$ in the presence of $\mathrm{ROH}$ and TMSOTf (wherein HOTf ${ }^{14}$ was produced in situ). ${ }^{15}$ An intramolecular nucleophilic addition of the anomeric oxygen onto

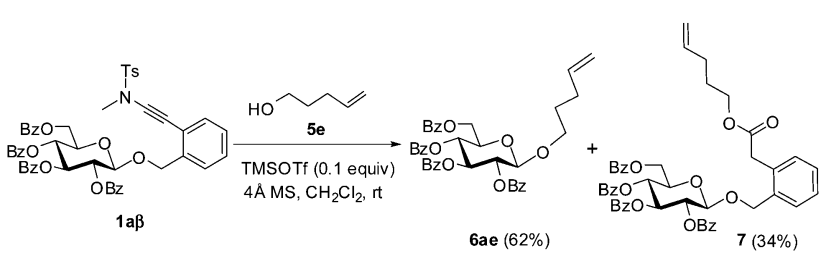

Scheme 2 The coupling of ortho-(methyltosylaminoethynyl)benzyl glucopyranoside 1a with 4-penten-1-ol (5e). 


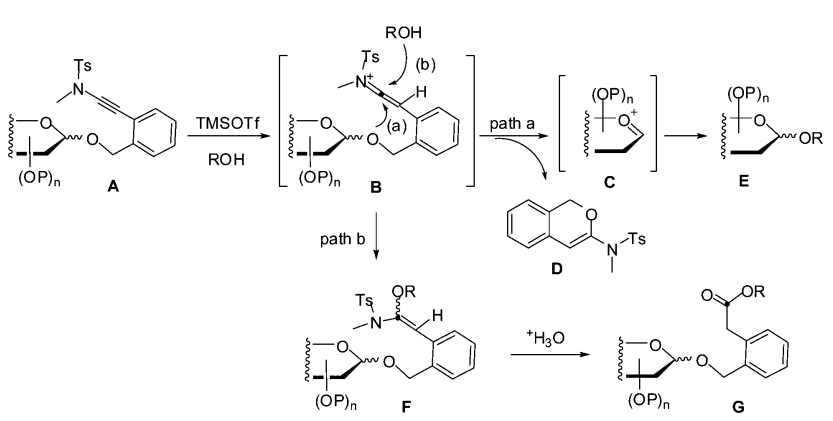

Scheme 3 A plausible mechanism for the glycosylation with ortho(methyltosylaminoethynyl)benzyl glycosides as donors under the catalysis of TMSOTf.

the keteniminium led to sugar oxocarbenium ion $\mathbf{C}$ and $1 H$-isochromene $\mathbf{D}$ which was indeed characterized (path a). Sugar oxocarbenium ion $\mathbf{C}$ underwent glycosylation in the presence of $\mathrm{ROH}$ to provide glycoside $\mathbf{E} .^{1 e}$ Alternatively, keteniminium cation $\mathbf{B}$ could be attacked by the alcohol $\mathrm{ROH}$, giving rise to alkoxysubstituted enamine intermediate $\mathbf{F}$ (path b). Hydrolysis of the enamine $\mathbf{F}$ during workup provided ester $\mathbf{G}$.

Given the fact that the ortho-(methyltosylaminoethynyl)benzyl glycoside donors are readily prepared from the orthoiodobenzyl glycosides, which are inactive in the glycosylation reactions of the former, these donors could be applied to the expeditious synthesis of oligosaccharides based on the 'latent-active' strategy. ${ }^{16}$ The previous donors applicable in the 'latent-active' synthesis of glycans include $p$-acetamidophenyl thioglycosides ( $v s . p$-nitrophenyl thioglycosides), ${ }^{17} n$-pentenyl glycosides (vs. 4,5-dibromopentyl glycosides), ${ }^{18}$ vinyl glycoside (vs. 1-methyl-2-propenyl glycosides), ${ }^{19}$ 2-(hydroxycarbonyl)benzyl glycosides (vs. 2-(benzyloxycarbonyl)benzyl glycosides), ${ }^{20}$ and $S$-benzimidazolyl glycosides ( $v s$. $N$-anisoylated $S$-benzimidazolyl glycosides). ${ }^{21}$ To demonstrate the feasibility of applying the present glycosylation protocol to the 'latent-active' assembly of glycans, the 'active' ortho-(methyltosylaminoethynyl)benzyl glycoside 1a was coupled with the 'latent' ortho-iodobenzyl glucoside derivative 8 in the presence of TMSOTf (0.1 eq.) to provide $\beta$ - $(1 \rightarrow 6)$-disaccharide $9(97 \%)$, which was then converted into the 'active' ortho-(methyltosylaminoethynyl)benzyl disaccharide 10 via Sonogashira coupling with ynamide 4 (88\%) (Scheme 4). Subsequent glycosylation of disaccharide 10 with cholesterol 5a or glucose-4-OH derivative 5c under similar glycosylation conditions furnished cholesterol 3-O- $\beta$-disaccharide 11 and $\beta$-trisaccharide 12 in $87 \%$ and $86 \%$ yields, respectively. In addition, glycosylation of disaccharide $\mathbf{1 0}$ with the 'latent' orthoiodobenzyl glucoside acceptor $\mathbf{8}$ provided the 'latent' orthoiodobenzyl trisaccharide $\mathbf{1 3}$ in $97 \%$ yield, which could be used for further elongation of the glycans via the iterative Sonogashira coupling/glycosylation sequence.

In conclusion, ortho-(methyltosylaminoethynyl)benzyl glycosides have been disclosed as a new type of glycosyl donors under the catalysis of TMSOTf. These shelf-stable donors are readily prepared from the corresponding ortho-iodobenzyl glycosides via Sonogashira coupling with ynamide 4. The expeditious assembly of glycans via the 'latent-active' strategy using the present protocol has been demonstrated. These
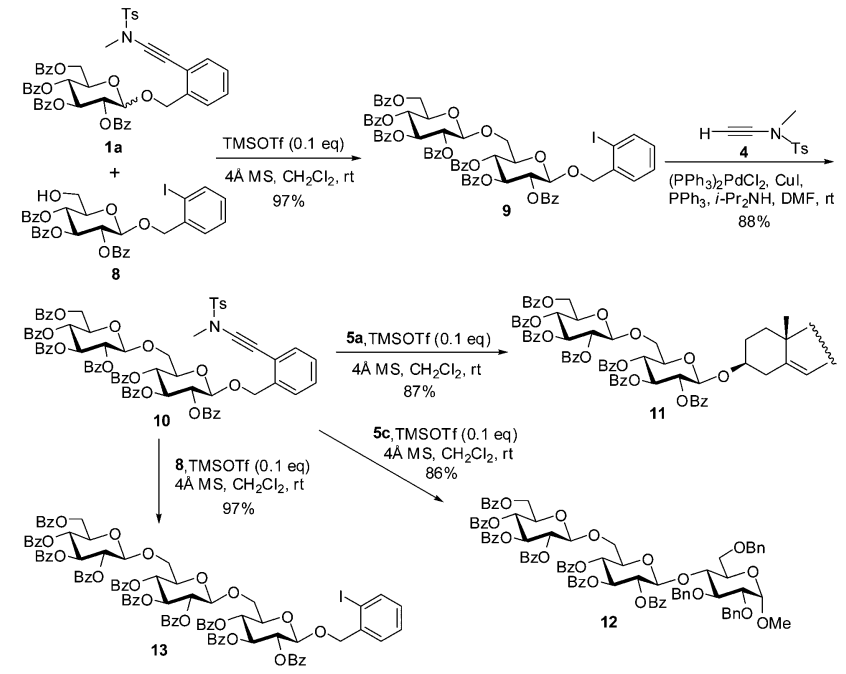

Scheme 4 Assembly of oligosaccharides 11-13 by the 'latent-active' strategy using ortho-(methyltosylaminoethynyl)benzyl glycosides as donors and ortho-iodobenzyl glycosides as acceptors.

promising preliminary results shall warrant further elaboration and application of this new glycosylation method.

Financial support from the Ministry of Science and Technology of China (2012ZX09502-002), the National Natural Science Foundation of China (21432012), the Fundamental Research Funds for the Central Universities (WY1514052), and the Shanghai Pujiang Program (15PJ1401500) is gratefully acknowledged.

\section{Notes and references}

1 (a) X. Zhu and R. R. Schmidt, Angew. Chem., Int. Ed., 2009, 48, 1900-1934; (b) M. J. McKay and H. M. Nguyen, ACS Catal., 2012, 2, 1563-1595; (c) B. Yu, J. Sun and X. Yang, Acc. Chem. Res., 2012, 45, 1227-1236; (d) S. S. Nigudkar and A. V. Demchenko, Chem. Sci., 2015, 6, 2687-2704; (e) L. Bohe and D. Crich, Carbohydr. Res., 2015, 403, 48-59; $(f)$ Y. Yang, X. Zhang and B. Yu, Nat. Prod. Rep., 2015, DOI: $10.1039 / \mathrm{C} 5 \mathrm{NP} 00033 \mathrm{E}$.

2 (a) S. Hotha and S. Kashyap, J. Am. Chem. Soc., 2006, 128, 9620-9621; (b) S. K. Mamidyala and M. G. Finn, J. Org. Chem., 2009, 74, 8417-8420; (c) S. R. Vidadala, S. A. Thadke and S. Hotha, J. Org. Chem., 2009, 74, 9233-9236; (d) S. R. Vidadala, G. Gayatri, G. N. Sastry and S. Hotha, Chem. Commun., 2011, 47, 9906-9908; (e) A. K. Kayastha and S. Hotha, Chem. Commun., 2012, 48, 7161-7163; $(f)$ A. K. Kayastha and S. Hotha, Beilstein J. Org. Chem., 2013, 9, 2147-2155.

3 (a) G. Sureshkumar and S. Hotha, Tetrahedron Lett., 2007, 48, 6564-6568; (b) G. Sureshkumara and S. Hotha, Chem. Commun., 2008, 4282-4284; (c) A. Y. Shaikh, G. Sureshkumar, D. Pati, S. S. Guptab and S. Hotha, Org. Biomol. Chem., 2011, 9, 5951-5959; (d) S. A. Thadke, B. Mishra and S. Hotha, Org. Lett., 2013, 15, 2466-2469; (e) S. A. Thadke, B. Mishra and S. Hotha, J. Org. Chem., 2014, 79, 7358-7371; $(f)$ B. V. Rao, S. Manmode and S. Hotha, J. Org. Chem., 2015, 80, 1499-1505.

$4 \mathrm{H}$. Imagawa, A. Kinoshita, T. Fukuyama, H. Yamamoto and M. Nishizawa, Tetrahedron Lett., 2006, 47, 4729-4731.

5 S. R. Koppolu, R. Niddana and R. Balamurugan, Org. Biomol. Chem., 2015, 13, 5094-5097.

6 (a) F. Yang, Q. Wang and B. Yu, Tetrahedron Lett., 2012, 53, 5231-5234; (b) S. Adhikari, K. N. Baryal, D. Zhu, X. Li and J. Zhu, ACS Catal., 2013, 3, 57-60.

7 (a) Y. Li, Y. Yang and B. Yu, Tetrahedron Lett., 2008, 49, 3604-3608; (b) Y. Li, X. Yang, Y. Liu, C. Zhu, Y. Yang and B. Yu, Chem. - Eur. J., 2010, 16, 1871-1882; (c) Y. Zhu and B. Yu, Angew. Chem., Int. Ed., 2011, 50, 8329-8332; (d) Y. Tang, J. Li, Y. Zhu, Y. Li and B. Yu, J. Am. 
Chem. Soc., 2013, 135, 18396-18405; (e) Y. Zhu and B. Yu, Chem. Eur. J., 2015, 21, 8771-8780.

8 (a) Y. Yang, Y. Li and B. Yu, J. Am. Chem. Soc., 2009, 131, 12076-12077; (b) Y. Li and B. Yu, Chem. Commun., 2010, 46, 6060-6062; (c) W. Yang, J. Sun, W. Lu, Y. Li, L. Shan, W. Han, W.-D. Zhang and B. Yu, J. Org. Chem., 2010, 75, 6879-6888; (d) Y. Ma, Z. Li, H. Shi, J. Zhang and B. Yu, J. Org. Chem., 2011, 76, 9748-9756; (e) Q. Zhang, J. Sun, Y. Zhu, F. Zhang and B. Yu, Angew. Chem., Int. Ed., 2011, 50, 4933-4936; $(f)$ Y. Li, J. Sun and B. Yu, Org. Lett., 2011, 13, 5508-5511; $(g)$ J. Yu, J. Sun and B. Yu, Org. Lett., 2012, 14, 4022-4025; (h) J. Zhang, H. Shi, Y. Ma and B. Yu, Chem. Commun., 2012, 48, 8679-8681; (i) J. Yu, J. Sun, Y. Niu, R. Li, J. Liao, F. Zhang and B. Yu, Chem. Sci., 2013, 4, 3899-3905; $(j)$ G. Xiao and B. Yu, Chem. - Eur. J., 2013, 19, 7708-7712; (k) S. Nie, W. Li and B. Yu, J. Am. Chem. Soc., 2014, 136, 4157-4160; (l) J. Li and B. Yu, Angew. Chem., Int. Ed., 2015, 54, 6618-6621; (m) X. Zhang, Y. Zhou, J. Zuo and B. Yu, Nat. Commun., 2015, 6, 5879, DOI: 10.1038/ncomms6879.

9 (a) C. A. Zificsak, J. A. Mulder, R. P. Hsung, C. Rameshkumar and L.-L. Wei, Tetrahedron, 2001, 57, 7575-7606; (b) G. Evano, A. Coste and K. Jouvin, Angew. Chem., Int. Ed., 2010, 49, 2840-2859.

10 (a) Y.-P. Wang and R. L. Danheis, Tetrahedron Lett., 2011, 52, 2111-2114; (b) M. R. Tracey, Y. Zhang, M. O. Frederick, J. A. Mulder and R. P. Hsung, Org. Lett., 2011, 13, 5508-5511.

11 (a) M. C. B. Jaimes, V. Weingand, F. Rominger and A. S. K. Hashmi, Chem. - Eur. J., 2013, 19, 12504-12511; (b) Y. Kong, K. Jiang, J. Cao, L. Fu, L. Yu, G. Lai, Y. Cui, Z. Hu and G. Wang, Org. Lett., 2013, 15, 422-425; $(c)$ T. Okitsu, K. Nakata, K. Nishigaki, N. Michioka,
M. Karatani and A. Wada, J. Org. Chem., 2014, 79, 5914-5920; (d) D. Fujino, H. Yorimitsu and A. Osuka, J. Am. Chem. Soc., 2014, 136, 6255-6258; (e) S. Kramer, K. Dooleweerdt, A. T. Lindhardt, M. Rottländer and T. Skrydstrup, Org. Lett., 2009, 11, 4208-4211.

12 D. Hou and T. L. Lowary, Carbohydr. Res., 2009, 344, 1911-1940.

13 E. Juaristi and G. Cuevas, The Anomeric Effect, CRC Press, 1994, pp. 183-194.

14 T. T. Dang, F. Boeck and L. Hintermann, J. Org. Chem., 2011, 76, 9353-9361.

15 Y. Zhang, R. P. Hsung, X. Zhang, J. Huang, B. W. Slafer and A. Davis, Org. Lett., 2005, 7, 1047-1050.

16 (a) T. C. Shiao and R. Roy, Top. Curr. Chem., 2011, 301, 69-108; (b) J. T. Smoot and A. V. Demchenko, Adv. Carbohydr. Chem. Biochem., 2009, 62, 161-250; (c) G. J. Boons, Tetrahedron, 1996, 52, 1095-1121.

17 (a) R. Roy, F. O. Andersson and M. Letellier, Tetrahedron Lett., 1992, 33, 6053-6056; (b) L. Huang, Z. Wang and X. Huang, Chem. Commun., 2004, 1960-1961.

18 B. Fraser-Reid, U. E. Udodong, Z. F. Wu, H. Ottosson, J. R. Merritt, C. S. Rao, C. Roberts and R. Madsen, Synlett, 1992, 927-942.

19 (a) G.-J. Boons and S. Isles, Tetrahedron Lett., 1994, 35, 3593-3596; (b) P. Wang, P. Haldar, Y. Wang and H. Hu, J. Org. Chem., 2007, 72, 5870-5873.

20 K. S. Kim, J. H. Kim, Y. J. Lee, Y. J. Lee and J. Park, J. Am. Chem. Soc., 2001, 123, 8477-8481.

21 S. J. Hasty, M. A. Kleine and A. V. Demchenko, Angew. Chem., Int. Ed., 2011, 50, 4197-4201. 